\title{
A Novel Method for Minimum Comprehensive Cost Network Coding in Wireless Network
}

\author{
Wu Yaohui ${ }^{\mathrm{a}}$ and Shao Pengfei ${ }^{\mathrm{b}}$ \\ School of Electronic and Information Engineering, Zhejiang Wanli \\ University, Ningbo 315100, China \\ ayaohuinb@aliyun.com, b56510005@qq.com
}

\begin{abstract}
Existing minimum cost network coding algorithms are basically optimized by a single indicator. This paper proposed a novel network coding method for minimum comprehensive cost, by the integration of multiple cost indicators. In the process of solving the problems by genetic method, the proposed algorithm realized the global optimal by the application of comprehensive cost indicator. Simulation results and analysis show that the proposed algorithm can significantly reduce the cost of network coding, while the computational complexity slightly increased.
\end{abstract}

Keywords: network coding, minimum cost, genetic algorithm.

\section{Introduction}

The concept of network coding was introduced in wireless network and immediately attracted a significant amount of attention from the research community [1, 2]. Among them, a large body of research focused on minimal cost network coding problem. SHEN XJ et al. [3] proposed an algorithm of network coding node selection based on super-key nodes by improving Ford-Fulkerson labeling algorithm. MINKYU K et al. [4] studied how to effectively design channel coding coefficient, and reduced the computational consideration by reducing the number of coding nodes or channels. Reducing the number of key links at the same time of constructing transmission paths is an effective way to minimize the additional cost of network coding, and a key links-based minimal cost network coding algorithm was proposed in [5]. The mathematic model had been created to estimate the computation cost of linear network coding and the relationship between operation cost and environmental parameters had been revealed in [6]. Hao Kun et al. [7] proposed an optimization algorithm for minimizing coding nodes, some new methods were introduced into the simple generic algorithm in order to avoid locality problem and to reduce optimization time.

For most of the minimal cost network coding problems addressed in the literature (e.g., [3]-[7]), the solutions optimized the target mostly based on a single 
indicator, such as minimum fee, minimum coding nodes, minimum coding links, minimum delay, or minimum operation cost etc. Someone proposed to re-optimization another indicator after optimizing one indicator. However it is not suitable for the application of the actual wireless network. In this paper, we first proposed a novel method for minimal comprehensive cost network coding in wireless network. According to the characteristics of the wireless network, we normalized the various indicators of wireless network into one comprehensive indicator, which was a synthesis of each specific weighted indicator, and the weighted coefficients can be flexibly adjusted according to the actual network by network executives.

\section{Problem Description}

Actually, all network nodes are not required to be participated in network coding in wireless network. To minimize the network coding cost, lots of literatures proposed a variety of optimized coding algorithm, but only based on a single indicator, such as minimum coding nodes etc. By analysis of the actual wireless network, it can be found that there are many network coding cost indicators. As shown in Fig. 1, if optimization algorithm is accordance with the minimum coding nodes, then Fig. 1(a) and Fig. 1(b) are both the final coding scheme. However, assuming the delay of link $\mathrm{AE}, \mathrm{CF}$ is 3,5 respectively, the fee of link AE, CF is 4, 2 respectively, the delay of network coding node E, F is 1,2 respectively, the fee of network coding node $\mathrm{E}, \mathrm{F}$ is 6,3 respectively. The joint optimization method with minimum coding nodes and minimum delay is shown in Fig. 1(a), minimum coding nodes and minimum fee is shown in Fig. 1(b). According to network coding cost indicators, solving the tradeoff between network coding cost and throughput maximization is a NP hard problem.

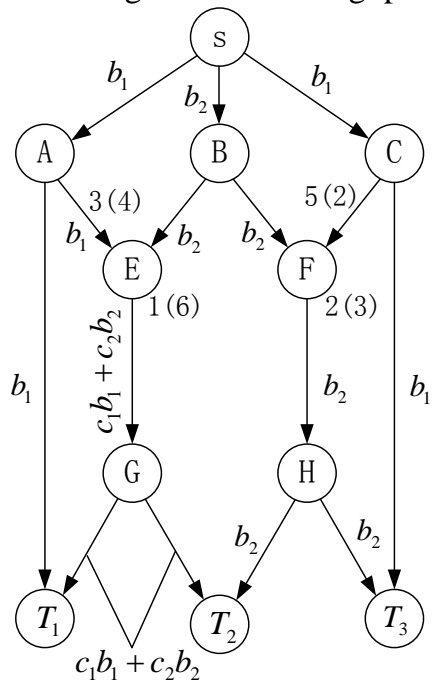

(a) minimum delay network coding

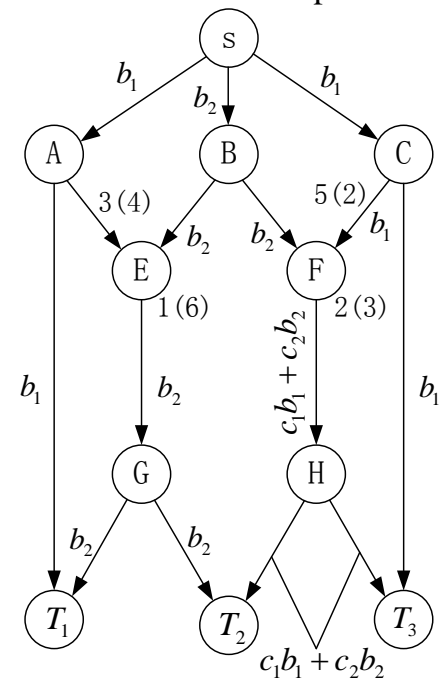

(b) minimum fee network coding 
Fig. 1 Network coding with multiple indicators

\section{Method}

1) Normalized indicator construction of network coding cost

If network coding algorithm is optimized by one indicator, it may cause the deterioration of other indicators. If simultaneously optimized by multiple indicators, it may cause logic conflict, and may affect the convergence of the algorithm at the same time. If re-optimized one indicator after another indicator optimized, the algorithm may be too complex, and it may be not suitable for the application of the actual wireless network. Therefore, normalized indicator construction of network coding comprehensive cost is particularly important. By network coding cost analysis, specific indicators are minimum fee, minimum coding nodes, minimum coding links, minimum delay, or minimum operation cost etc. These indicators should be normalized into one indicator, which is so-called comprehensive cost. Assuming the fee, number of coding nodes, number of coding links, delay, operation cost of the i-th node is $F_{i}, N_{i}, L_{i}, T_{i}, O_{i}$ respectively, the weight value is $a_{1}, a_{2}, a_{3}, a_{4}, a_{5}$ respectively, the indicator adjustment coefficient is $q_{1 i}, q_{2 i}, q_{3 i}, q_{4 i}, q_{5 i}$ respectively, the comprehensive cost is $\mathrm{P}$. Define $\mathrm{P}$ as equation 1.

$$
P=\sum\left(a_{1} q_{1 i} F_{i}+a_{2} q_{2 i} N_{i}+a_{3} q_{3 i} L_{i}+a_{4} q_{4 i} T_{i}+a_{5} q_{5 i} O_{i}\right)
$$

The weight values satisfy the equation 2 .

$$
a_{1}+a_{2}+a_{3}+a_{4}+a_{5}=1
$$

The weight values are constant that can be determined in advance according to the focused network coding cost. For example, if $a_{2}$ is one and other weight values are zero, then current comprehensive cost is number of coding nodes. The indicator values are also constant that can be determined in advance according to the network topology. For example, if one node is coded, then the number of coding nodes will be added 1 , the fee will be added statistical average fee of the node. The indicator adjustment coefficient will be changed in the coding algorithm execution. For example, when coding route first pass the i-th coding node, $q_{1 i}$ and $q_{5 i}$ will be both one, which means that the comprehensive cost will only be added fee and operation cost. When coding route second pass the i-th coding node, $q_{1 i}$ will be zero and $q_{5 i}$ will be less than one, which means that the comprehensive cost will only be added discounted operation cost. It can be seen that the strategy implies minimum fee, minimum coding nodes and minimum operation cost.

2) Combination comprehensive cost with genetic algorithm 
Random network coding is the ideal coding scheme of wireless network. It is a NP hard problem, we solve it by combining comprehensive cost with genetic algorithm. The genetic algorithm is an intelligent search method, it must search in the whole problem space. The problem space is feasible coding scheme for all multicast rate $\mathrm{C}$, the solving target is to search out a coding scheme which is workable, multicast rate $\mathrm{C}$, and minimum comprehensive in all possible coding scheme collection. Testing random network coding method with multicast rate $\mathrm{C}$, which is equivalent of a search process. Fitness evaluation is needed for search point in the search process. Fitness evaluation need two indicators, namely, whether the coding scheme is feasible, the required comprehensive cost. In the genetic algorithm combined with comprehensive consideration, we first convert the problem space into the coding space, and then search for feasible coding scheme, calculate the desired comprehensive cost, finally obtain possible coding scheme with minimum comprehensive cost.

3) Construction of coding instructions template in genetic algorithm

In genetic search process, the traditional random coding scheme operates template coding instruction of each channel through the cross and the mutation operation to. However, we use comprehensive cost to control the cross and the mutation operation, and finally decide the probability that the coding instruction template component value is 1 . It can be seen in Fig. 2, once coding instruction template is decided, if the value is 0 , then the corresponding local coding vector is 0 , otherwise it is a random number in the range of $G F\left(2^{m}\right)$.
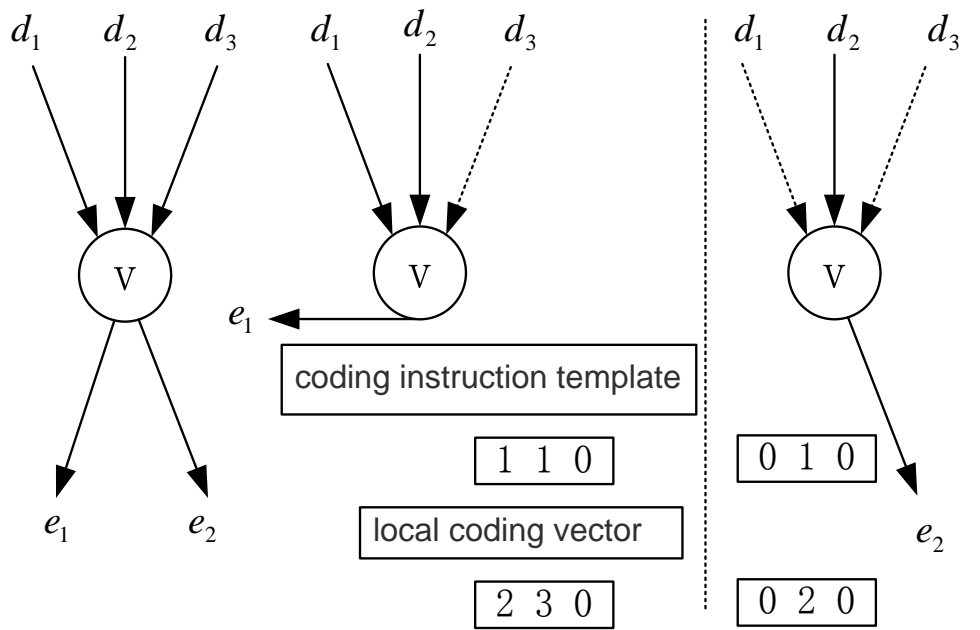

Fig. 2 Coding instruction template and local coding vector

If a node does not have the function of network coding, the coding instructions template vector will be zero. In addition, we introduce strong genes and weak gene in the process of cross and mutation. If one node or one link can significantly improve comprehensive cost, then it will be set with a strong gene. If 
it can deteriorate comprehensive cost, then it will be set with a weak gene. Otherwise, it will be set with a normal gene.

\section{Algorithm Implementation}

This algorithm is of based on standard genetic algorithm, the specific process is as follows:

1) Population initialization and adaptation function. According to all of the outside edges of the optimization nodes, make chromosome's structure. Assume the number of the optimization nodes in the graph $G$ is $N$, the length of each chromosome is $\sum_{i=1}^{N} \operatorname{In}\left(v_{i}\right) \operatorname{Out}\left(v_{i}\right)$. Assume each network coding cost indicator is randomly generated according to the network topology, the weight and the adjustment coefficient is 0.2 and 1 respectively. For chromosome $k$, the comprehensive cost is $E_{k}$, the fitness function is $1 / E_{k}$.

2) Intersection and Mutation. According to the fitness function, randomly select two intersection points, and then randomly select a number from the set $\{0,1,2\}$. If the number is 0 , exchange with the chromosome before the first intersection point. If the number is 1 , exchange the chromosome between the two intersection points. If the number is 2 , exchange with the chromosome after the second intersection point. According to the indicator adjustment coefficient, select some coding coefficient in the chromosome as the mutation point, replace with new coding coefficient after random variation, then form a new chromosome.

3) The acceptance strategy of the new individual. Solve the fitness function of the new individual generated in the genetic operation, calculate the comprehensive cost according to the formula (1), then select the corresponding acceptance strategy, that is to accept the new individual with the probability value $p$.

4) Duplicate. Select and reproduce the optimal individual of the population, then join the new individual into new population.

5) Judge whether the algorithm terminates. According to the current new population, calculate the comprehensive cost and the network throughput. If the termination condition is not satisfied, then return to step 2; otherwise, the algorithm terminates.

\section{Simulation and Analysis}

Wireless access network is simulated through the software Brite [8]. Randomly generated network topology is represented by four-tuple, i.e. rate, number of nodes, links, and destination nodes. Assume each cost indicator is randomly generated according to the network topology, the weight and the adjustment coefficient is 0.2 and 1 respectively. The population size is 200 , the 
intersection probability is 0.90 , the rate of variation is 0.01 , and the maximum algebra is 500 .

Table 1 shows the comparison of simulation results between the proposed algorithm and literature [7]'s algorithm. As can be seen, at the same number of nodes, the comprehensive cost of the proposed algorithm is significantly less than literature [7]'s algorithm. The gap is more obvious especially when the number of the network nodes increased. This is because the proposed algorithm is to carry on network coding according to multiple cost indicators, while literature [7]'s algorithm done only according to single indicator, that is the number of coding links. At the same number of nodes, the optimization time of the proposed algorithm is slightly longer than the literature [7]'s algorithm, it shows that the proposed algorithm increases the computational complexity in the integration of multiple cost indicators, but in the acceptable range.

Table 1 Comparison of simulation results

\begin{tabular}{ccccc}
\hline \multirow{2}{*}{ Number of nods } & \multicolumn{2}{c}{ Proposed } & \multicolumn{2}{c}{ Literature [7] } \\
\cline { 2 - 5 } & Cost & Opt. Time(s) & Cost & Opt. Time (s) \\
\hline 10 & 6 & 14 & 9 & 11 \\
20 & 14 & 170 & 18 & 150 \\
30 & 18 & 490 & 27 & 430 \\
40 & 22 & 980 & 36 & 900 \\
50 & 25 & 1700 & 43 & 1600 \\
\hline
\end{tabular}

In order to analysis and evaluate the convergence of the proposed algorithm, figure 3 compares the average fitness of the population in two algorithms when the number of nodes is 20 . As can be seen, the proposed algorithm converges to a global optimal solution, the average fitness value is significantly higher than literature [7]'s algorithm, this is because the proposed algorithm considers multiple cost indicators. The proposed algorithm's fitness value keeps stable after 250 generations, slightly more than 220 generations of literature [7]'s algorithm, it shows that considering multiple indicators slows the convergence rate of the proposed algorithm. 


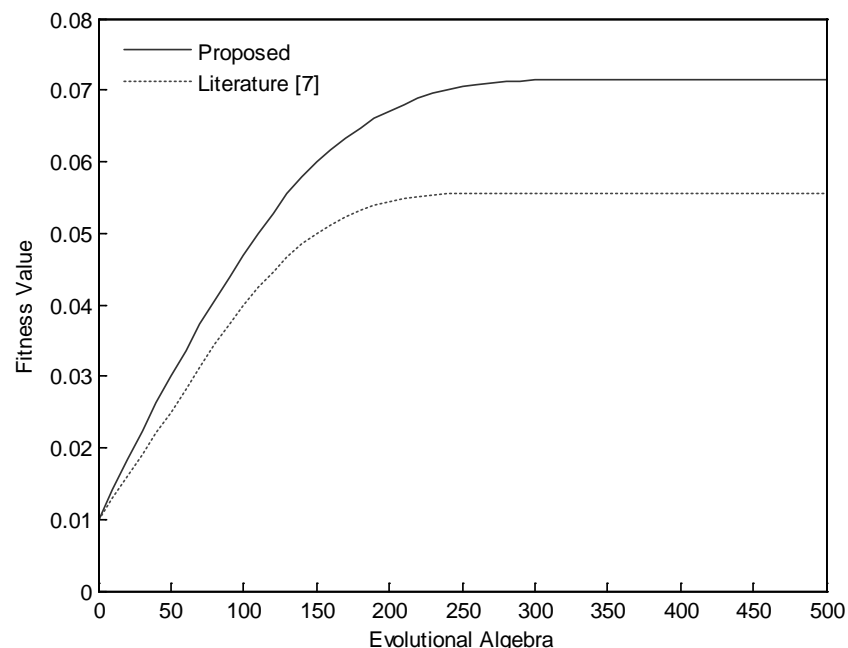

Fig. 3 Comparison of the population's average fitness

\section{Conclusion}

In view of the existence of multiple network coding cost indicators in wireless network, this paper proposes a network coding method of minimum comprehensive cost. In the process of solving the problems by genetic method, do intersection and mutation, according to the corresponding comprehensive cost, realizes the global optimal. The simulation results show that the network coding cost is reduced obviously, while the computational complexity slightly increased.

\section{Acknowledgements}

This work was supported by Zhejiang Provincial Natural Science Foundation (LY14F010007), the Ningbo Natural Science Foundation (2012A610061, 2012A610016).

\section{References}

[1] AHLSWEDE R, CAI N, LI S R, et al. Network information flow[J]. IEEE Transactions on Information Theory, 2000, 46(4): 1204-1216.

[2] HO T, MEDARD M, KOETTER R, et al. A random linear network coding approach to multicast[J]. IEEE Transactions on Information Theory, 2006, 52(10): 4413-4430. 
[3] SHEN Xiao-jian, CHEN Zhi-gang, et al. Analysis of Network Coding Nodes Selection in Wireless Mesh Network [J]. Computer Science, 2010, 37(10): 71-74.

[4] MINKYU K. Evolutionary approaches to minimizing network coding resources[A]. 26th IEEE International Conference on Computer Communications[C]. Anchoge, AK, 2007: 1991-1999.

[5] TAO S G, HUANG J Get al. An improved algorithm for minimal cost network coding[J]. Journal of Huazhong University of Science and Technology, 2008, 36(5): 1-4.

[6] PU Bao-xing, WANG Wei-ping. Evaluation and analysis of the computation cost of linear network coding[J]. Journal on Communications, 2011, 32(5):47-55.

[7] HAO Kun, JIN Zhigang. An optimization algorithm of network coding for minimizing coding nodes[J]. Journal of Electronics \& Information Technology, 2011, 33( 2) : 260-265.

[8] BRITE. Overview of Brite [EB /OL]. http://www.cs.bu.edu/brite/index.html. 